\title{
Adequacy Analysis and Security Reliability Evaluation of Bulk Power System
}

\author{
Okwe Gerald Ibe, Inyama Kelechi \\ Department of Electrical/Electronic Engineering Federal University of Technology Owerri Imo State \\ (NIGERIA)
}

\begin{abstract}
Since the advent and integration of information technology into power industry, the complexity of modern power systems and advances in the power industry toward restructuring, the system models and algorithms of traditional reliability assessment techniques are becoming obsolete as they suffer from nonrealistic system models and slow convergence (even non-convergence) when multi-level contingencies are considered and the system is overstressed. To allow more rigor in system modeling and higher computational efficiency in reliability evaluation procedures, this paper proposes an analytically-based security-constrained adequacy evaluation (SCAE) methodology that performs bulk power system reliability assessment and gave an in depth analysis of the concept of the state of the art in bulk power system reliability assessment. Bulk power system reliability assessment is an important procedure at both power system planning and operating stages to assure reliable and acceptable electricity service to customers.

Keywords: Bulk power system reliability, Adequacy, Contingency, Monte Carlo simulation, quality and security.
\end{abstract}

\subsection{Bulk Power System Reliability}

\section{Introduction}

An electric power system is generally composed of three parts: (1) generation, (2) transmission, and (3) distribution systems, all of which contribute to the production and transportation of electric energy to consumers. The reliability of an electric power system is defined as the probability that the power system will perform the function of delivering electric energy to consumers on a continuous basis and with acceptable service quality [1]. For the purpose of conducting power system reliability assessment, the three power system parts are combined into different system hierarchical levels, as shown in Figure 1.1 $[2,3]$. Hierarchical level 1 (HL1) involves the reliability analysis of only the generation system, hierarchical level 2 (HL2) includes the reliability evaluation of the composite of both generation and transmission systems, referred to as the bulk power system or the composite power system, and hierarchical level 3 (HL3) consists of a reliability study of the entire power system.

At the present stage of development, the reliability evaluation of the entire power system (HL3) is usually not conducted because of the immensity and complexity of the problem in a practical system. Instead, power system reliability is assessed separately for the generation system (HL1), the bulk power system (HL2), and the distribution system [4]. Reliability analysis methods for generation and distribution systems are well developed due to difficulties arising from the huge computational burden associated with the bulk power system reliability analysis [1]. Thus, this research concentrates on the area of bulk power system reliability assessment.

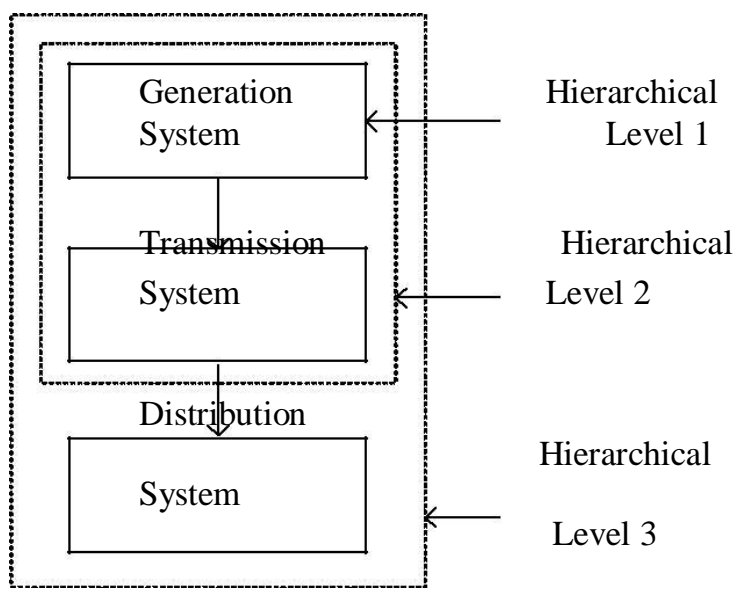

Figure 1.1: Hierarchical levels for power system reliability assessment. 
Bulk power system reliability assessment refers to the process of estimating the ability of the system to simultaneously (a) generate and (b) move energy to load supply points [4]. Traditionally, it has formed an important element of both power system planning and operating procedures.

1.2 The main objective of power system planning: This is to achieve the least costly design with acceptable system reliability. For this purpose, long-term reliability evaluation is usually executed to assist long-range system planning in the following aspects: (1) the determination of whether the system has sufficient capacity to meet system load demands, (2) the development of a suitable transmission network to transfer generated energy to consumers load points, (3) a comparative evaluation of expansion plans, and (4) a review of maintenance schedules [5, 6].Power system operating conditions are subject to changes such as load uncertainty, i.e., the load may be different from that assumed in design studies, and unplanned component outages. To deliver electricity with acceptable quality to customers at minimum cost and to prevent cascading sequences after possible disturbances, short-term reliability prediction that assists operators in day-to-day operating decisions is needed. These decisions include determining short-term operating reserves and maintenance schedules, adding additional control aids and short lead-time equipment, and utilizing special protection systems [7, 8].The SCAE methodology adopts a single-phase quadratized power flow (SPQPF) model as a basis and encompasses three main steps: (1) critical contingency selection, (2) effects analysis, and (3) reliability index computations. In the critical contingency selection, an improved contingency selection method is developed using a wind-chime contingency enumeration scheme and a performance index approach based on the system state linearization technique, which can rank critical contingencies with high accuracy and efficiency. In the effects analysis for selected critical contingencies, a non-divergent optimal quadratized power flow (NDOQPF) algorithm is developed to (1) incorporate major system operating practices, security constraints, and remedial actions in a constrained optimization problem and (2) guarantee convergence and provide a solution under all conditions. This algorithm is also capable of efficiently solving the ISO/RTO operational mode in deregulated power systems. Based on the results of the effects analysis, reliability indices that provide a quantitative indication of the system reliability level are computed. In addition, this research extends the proposed SCAE framework to include the effects of protection system hidden failures on bulk power system reliability. The overall SCAE methodology is implemented and applied to IEEE reliability test systems, and evaluation results demonstrate the expected features of proposed advanced techniques.

\subsection{Bulk Power System Reliability Modeling Methods}

Bulk power system reliability modeling techniques have evolved from traditional deterministic modeling methods to the current more advanced probabilistic modeling methods. Recently, some intelligent concepts, such as fuzzy set theory, have also been incorporated into probabilistic modeling techniques.

\subsubsection{Deterministic Method}

It is incumbent on power system planners and operators to ensure that customers receive adequate and secure energy supplies within reasonable economic constraints. Historically, such a task has involved the assessment of bulk power system reliability using deterministic criteria [18, 19], which generally include a list of empirical contingencies involving the outages of some important power system components. With these contingencies in mind, planners and operators of the power system can incorporate sufficient redundancy so that any system failures during such contingencies can be prevented. The more comprehensive the list of contingencies, the lower the probability of a system failure resulting from contingencies not listed. Through such deterministic methods, a satisfactory degree of system reliability has been achieved in the past decades [4].It is crucial that power system planners more closely examine the key element of deterministic criteria, i.e., the contingency list. The contingency list, which is generally developed based on the planners' experience, is often the result of an informal framework [4]. As power systems become more complex, and unforeseen power flow patterns occur because of independent power producers, the contingency list becomes a less reliable means of assessing the power system reliability because the impact of contingencies absent from the list may become significant [20]. Therefore, the trend is to develop automated procedures for selecting the contingency list by taking into account power flow patterns and new operating scenarios.

\subsubsection{Probabilistic Method}

As power systems grow much larger and more complex, the need for the rigorous analysis of bulk power system reliability becomes more obvious. Because of the stochastic nature of system behavior, such as component outages or load-level changes, the development and application of probabilistic techniques for modeling the bulk power system and evaluating an appropriate set of expected risk indices have received considerable attention [2-4]. In the probabilistic modeling method, uncertainties affecting power system reliability are accounted for using probabilistic techniques. For example, the Markov model is widely used to 
represent component states, electric load levels, system states, and so on. Such a probabilistic modeling method enables the calculation of probability, frequency, and duration indices of system failures. These indices are no longer deterministic values but expected values of probability distributions $[2,3]$.

\subsubsection{Fuzzy-Probabilistic Method}

In recent years, some power system reliability probabilistic modeling methods have attempted to incorporate the fuzzy set concept in modeling system uncertainties [21-26]. For example, fuzzy numbers are used to model uncertainties in system component failure and repair rates, fuzzy load duration curves are developed using a fuzzy number in each time step [21-24], and fuzzy power flow models [25, 26] are developed to identify possible system behaviors given specified uncertainties. These fuzzy representations of system uncertainties are then integrated into the probabilistic evaluation procedure. However, by using this fuzzyprobabilistic method, the computational burden increases significantly without a commensurate gain in the quality of results.

\subsection{Bulk Power System Reliability Assessment Methods}

Current bulk power system reliability assessment techniques in the literature fall into two fundamental categories: analytical and Monte Carlo simulation techniques [27-31].

\subsubsection{Analytical and Monte Carlo Simulation Techniques}

The reliability assessment of bulk power systems must consider a multiplicity of factors, such as the failure and repair rates of equipment and operating practices, including economic generation scheduling, security controls, emergency controls, projected load variations, and maintenance schedules. These system characteristics can be represented with analytical models in the analytical technique [2, 4, and 32]. By using the analytical technique, system contingencies, which may involve line failures, unit outages, or both, are first enumerated up to a certain level. To identify the contingencies that result in system failures, failure effects analysis is then conducted to test system contingencies against some predetermined criteria. The impact of each contingency on the system, such as line loading and bus voltages, is obtained by solving power flows. Based on the results of effects analysis, system reliability indices such as loss-of-load probabilities, frequencies, and durations can be calculated $[33,34]$.The bulk power system reliability assessment problem is treated as a series of experiments by the Monte Carlo simulation technique. This technique consists of randomly sampling system states, testing them for acceptability, and aggregating the contribution of loss-of-load states to the reliability until the variations of reliability indices drop below pre-specified tolerances [3]. The basic approach can be applied for each hour in a year in chronological order (the sequential approach), or the hours of the study time can be considered as random (the random approach). The simulation of selected system states is done with the use of load flows that consider generation dispatch and other pre-selected operating policies. Simulation results are distributions of the variables of interest (i.e., circuit flows, voltage levels, energy curtailment, and so on). These results are utilized in the computation of appropriate reliability indices [35-37].

\subsubsection{The Comparison of Two Techniques}

The comparison of analytical and Monte Carlo simulation techniques is performed in this section. Both techniques solve power flow problems to identify system deficiencies. Power flow models vary widely and differ in computing speed, precision, and computer storage requirement. Since the number of contingencies in a practical system is enormous, a large number of power flow problems have to be solved. Therefore, in both analytical and Monte Carlo simulation techniques, the employed power flow model plays an important role in assessing overall system reliability efficiently and accurately [4]. On the other hand, the analytical and the Monte Carlo simulation techniques differ with regard to the processes of selecting states and evaluating reliability indices. The analytical technique selects states in an increasing order in terms of contingency levels, and this process stops at a particular component outage level or when the state probability becomes less than a specified value. Each state is assessed only once, and reliability indices are calculated mathematically from the statistical data associated with each state. Monte Carlo simulation techniques, however, select states randomly based on the concept of random numbers. States with greater probabilities of occurrence are more likely to be simulated several times, and this simulation process stops either after a fixed number of simulations or on the basis of statistical stopping rules. The expected values of indices are determined by averaging the indices obtained from each simulation [3].

In Monte Carlo simulation, the number of system states that must be sampled before the indices stabilize is extremely large, and this number increases significantly as the system reliability level increases. Thus, a major drawback of the Monte Carlo simulation method is that it requires a long computation time. In particular, when using the sequential approach, the computational burden may be unmanageable for a practical bulk power system. Besides the long computation time, another limitation exists in the random approach. In 
power systems, the outages of some components may influence the probabilities of related component outages, which are referred to as "fat tail" effects. Since the random approach does not include this sequence information, it cannot account for the fat tail effects in the simulation procedure. Compared to Monte Carlo simulation, the analytical technique can provide reliability indices in a relatively short computation time. It can also consider fat tail effects and other complex processes of the system using appropriate analytical models without significantly increasing the computational burden. In addition, the computational effort in the analytical method is much less dependent on the system reliability level.

\subsection{Adequacy and Security Reliability Assessment}

Bulk power systems must not only provide adequate generation and transmission capacity to meet the demand of customers while satisfying system operating constraints, but also be capable of maintaining system stability following faults, switchings, and other disturbances. Regarding these two aspects, the reliability of a bulk system can be evaluated by two attributes: adequacy and security [2, 39].

Adequacy refers to the ability of the system to supply the aggregate electric energy requirements of customers within component ratings and voltage limits when planned and unplanned component outages occur. Adequacy assessment involves system steady-state conditions of post-contingencies, i.e., the system is assumed to always reach a stable equilibrium point after equipment outages, and the dynamics of the transition from one state to another are neglected. The second attribute, system security, refers to the ability of the power system to withstand disturbances arising from faults or equipment outages. Security assessment involves system transient responses and cascading sequences after a disturbance. Transient responses include the fluctuations of both the system frequency and bus voltages. If the fluctuations exceed certain operating limits, cascading sequences, such as line and generator tripping, may occur and persist until the system completely separates or collapses. These effects may not be properly accounted for in adequacy studies and must be captured in security evaluations. Although most research has been devoted to the adequacy assessment of bulk power system reliability, some concerns have recently shifted to security evaluations that also integrate the adequacy studies. The following two sections summarize the main approaches developed in these two areas.

\subsubsection{Adequacy Assessment}

The major difficulty in adequacy assessment involves the enormous computational effort required to analyze as many contingencies that may have a nonzero contribution to system unreliability as possible. The massive computational demand is a result of the following two factors: The first involves the large system size and the resulting large number of system states that must be assessed. It is usually not feasible or even possible to investigate all the contingencies of a power network. In practice, only credible outage states up to a certain contingency level are investigated. It has been noticed that as the system size increases, the consideration of higher-level contingencies is necessary, which will rapidly increase the computation time [4]. Another factor involves the complexity of the failure effects analysis in the assessment of each state. The objective of such an analysis is to identify the impact of each state on system operations by solving power flow problems. It is the most important but the most computationally demanding part of reliability assessment, and its performance influences the effectiveness of the overall reliability evaluation [2, 4].Research attempting to solve the above two problems are further classified as adequacy and security-constrained adequacy evaluations, which are reviewed separately as follows.

\subsubsection{Adequacy Evaluation}

The following research effort based on analytical techniques has been dedicated to reducing the number of contingencies that must be evaluated and therefore to cut down the computational burden while at the same time increasing the assessment accuracy. Inclusion of State Effects without Actual Evaluation To improve reliability assessment, a state extension algorithm is proposed [40,41]. In this method, the system state space is enumerated in the form of a tree graph based on different outage levels, and a coherent system is assumed, i.e., if an investigated state is a failure state, the descendant states with higher outage levels in the sub-tree of this investigated state are concluded to be failures, too. The information of the sub-tree, including its probability and associated repair and failure rates, is computed based on the knowledge of the investigated state. The contribution of states in the sub-tree to system unreliability is taken into account when system reliability indices are calculated. In this method, the effects of some un-investigated high-level contingencies are included in the reliability evaluation procedure, and more accurate indices are obtained without increasing the computational burden. The drawback of this algorithm is that it requires the component sequence adjustment in the tree graph so that it includes as many effects as possible of un-investigated high-level failure states. In its application to the testing systems [40], results show that it could not provide satisfactory assessment in some situations. In addition, for a large system, even the number of contingencies up to the boundary outage level is too large to be examined one by one [40]. A possible solution is to use the contingency selection scheme, which provides 
information useful in adjusting the component sequence to further reduce the computational effort in evaluating the states up to the boundary outage level.

Simplification of the System Model by System Equivalent Models The practical bulk power system can be extremely complex because of the large number of components and complex interconnections among them. To simplify the system model, an adequacy equivalent method $[42,43]$ is proposed, in which a general power network is divided into two areas: the area of interest (AI) and the interconnected area (IA). The IA is replaced with an equivalent model that is simpler than the original network but retains all the essential information. The equivalent model is expressed in terms of a capacity margin table that contains the information of available capacities, probabilities, and frequencies of simplified states in the IA. Once this equivalence is developed, the adequacy evaluation can be conducted effectively with reduced computational effort. In [44, 45], the equivalent method is extended to include the equivalent load model in the IA so that its load curtailment can also be considered. Furthermore, in [46], the composite power system is divided into three areas: the area of interest, the boundary buses, and the external area. The external area is replaced by an equivalent probabilistic model, and the statistical behavior of its components is included. Although the system equivalent method can simplify the analysis to some extent and reduce the computational burden, it should be noted that this method has some limitations. First, an equivalent model is a simplified model of the original network and cannot complete and precise in all aspects. In addition, this method is system dependent, i.e., different equivalent models need to be developed under different system operating conditions and for different areas of interest. As a result, the extra computational effort is brought into the evaluation procedure. Furthermore, the implementation of this method is based on the DC power flow model, which cannot check the voltage and reactive power of the power supply at load points and capture the effects of voltage and reactive power supplies on system reliability Reduction of State Space by the Contingency Selection Method In adequacy assessment, an important fact is that many component outages (contingencies) do not cause the service curtailment or other adverse impact on service quality [47-49]. The contingency selection method can eliminate states whose impact on the system is small, thus reducing the state space to include only states whose impact on the system is significant. Selected states are further ranked according to their levels of impact on the system [52]. Typical contingency selection methods consist of the performance index (PI) method, the screening method, and the hybrid scheme.

In the PI method, a variety of performance indices, such as the circuit current index, the voltage index, and the reactive power index, are defined to measure the normality of a system [51-55]. When a contingency occurs, the system operating condition changes and so do the associated PIs. The variations of PIs from precontingency to post-contingency can be considered as an indication of the impact of the contingency on system operations. Contingencies are further ranked in a descending order of the projected PI changes. Although the PI method is efficient, it is vulnerable to misrankings. The inaccuracies of the PI method are mainly the result of (1) the nonlinearities of the system model [55] and (2) the discontinuities of the system model arising from generator reactive power limits, regulator tap limits, and so on $[53,54]$. In contrast to the PI method, the screening method is more accurate but inefficient. In this method, the contingency selection is based on approximate network solutions such as Fast Decoupled Power Flow solutions [54, 56], which can consider the nonlinearities of the power system to some extent and provide more accurate results than the PI method. However, the contingency selection using the screening method needs to solve post-contingency cases, which are time consuming. In particular, time may be wasted while solving contingencies without much impact on system operations. The hybrid scheme [5, 50], a combination the PI and the screening methods, achieves both efficient and accurate contingency selection and ranking. Efficiency is achieved by employing the PI method first to quickly identify the conceivable contingencies. The accuracy of ranking is guaranteed by using screening methods for only a subset of contingencies that cannot be ranked with confidence by the PI methods. Thus, the hybrid scheme employs the best properties of both the PI and the screening methods.

\subsubsection{Security-Constrained Adequacy Evaluation (SCAE)}

In the previous section, the main research effort focused on developing techniques that select credible contingencies. As far as effects analysis is concerned, traditional power flow techniques, including DC power flow and AC power flow, are generally used as the primary means to perform contingency evaluations. Some recent research efforts have concentrated on improving effects analysis performance by integrating remedial/corrective actions, i.e., remedial actions are applied to satisfy system security constraints while solving power flow problems after contingencies. This strategy is referred to as security-constrained adequacy evaluation SCAE). System security constraints include bus voltage limits, line flow limits, real power and reactive power generation limits, and so on. In practice, when generator or line outages occur or line overloads resulting from outages occur generating units should be rescheduled so that the power system can maintain generation-demand balance and alleviate line overloads. If violations corresponding to the bus voltage limits exist, reactive power (generation, transformer taps, and shunt compensation) must be rescheduled to eliminate abnormal voltages in the system. The purpose of these remedial actions is to keep the system operating 
normally, and thus avoid load curtailment, if possible, or to minimize total load curtailment, if unavoidable. In some security-constrained adequacy evaluation methodologies are presented. In these methods, the procedures for effects analysis are implemented by solving power flow problems coupled with remedial actions. Optimization power flow problems are formulated to minimize the load curtailment through remedial actions and load shedding in the effects analysis procedure. Linear programming models for remedial actions that adjust active power and reactive power are derived. Some techniques, including the simplex method and the interior point method are applied to solve the formulated optimal power flow problems. Since the optimal power flow technique is an important issue related to the effects analysis procedure in SCAE, a brief review of the formulation and the solution of optimal power flow is provided in the following section.

\subsubsection{Optimal Power Flow}

Optimal power flow (OPF) is a mathematical optimization tool for adjusting the power flow in a power network to achieve the optimal value of a predefined objective while satisfying system operating constraints. OPF has undergone intensive research and development over the past several decades . Mathematically, the general OPF problem can be expressed as follows.

Minimize $f(x, u)$

Subject to $g(x, u)=0$

$$
h(x, u) \leq 0
$$

${ }_{\text {where }} \min _{\leq u \leq u} \max$,

$f(x, u)$ objective function

$g(x, u)=0$ power flow equations

$h(x, u) \leq 0$ operating constraints $x$ vector of

system state variables $u$ vector of control

variables.

$u^{\min } \leq u \leq u^{\max }$ feasible region of control variables.

The OPF objective function varies with different operational objectives. Typical examples of objective functions are minimum generation cost minimum system transmission loss, voltage and reactive power optimization, preventive and corrective control optimization, and so on. In reliability assessment, the objective may include the minimum amount of remedial actions, minimum load curtailments, and so on. A predefined objective can be achieved if control variables in the system are available for adjustment. Control variables usually include MW/MVAR generation adjustment; shunt capacitor/reactor switching, phase shifter adjustment, transformer tap adjustment, load transfer, area interchange, and load shedding.

Constraints in the OPF generally contain equality and inequality equations. Equality constraints are usually power flow equations. Inequality constraints consist of functional operating constraints, including branch flow limits, bus voltage magnitude limits, and so on. In addition, the feasible region of control variables is contained in the constraints set, including unit active and reactive power output limits, transformer tap limits, and so on. Some variations in formulating the OPF problem exist with its application in different areas. These variations mainly include the decomposition of real and reactive OPF, contingency constrained OPF, stability constrained OPF, and so on. Also, a wide variety of optimization techniques, such as linear programming (LP), quadratic programming, nonlinear programming, hybrid versions of linear programming, and integer programming, have been employed to solve the formulated OPF problems. Because of the large-scale nature of the problem and the resulting computational complexity of reliability assessment, the linear programming (LP) technique, which has a relatively simple formulation and is capable of providing fast solutions, is the most attractive tool.

The application of the LP technique requires that the nonlinear objective function and constraints be linearized around the operating point. The obtained LP problem can be solved using the different variants of the simplex method. Among them, the most commonly used technique is the revised simplex method. However, interior point methods have recently shown superiority over the simplex method for solving large-scale LP problems.

In spite of the tremendous research that has been done, the OPF remains a difficult mathematical problem, and its use has been somewhat restricted by its lack of efficiency, flexibility, and reliability. To solve such problems, a novel formulation and solution approach for the OPF problem was developed. The optimal solution is obtained by gradually reducing the power mismatch at each bus while at the same time incorporating the economic dispatch process and observing operating constraints. This OPF model has the following merits: (1) it guarantees a non-divergent solution if a feasible power flow solution exists; (2) it realistically simulates actual system operating practices by incorporating the economic dispatch and remedial actions when operating 
constraint violations occur; and (3) compared with other OPF models, it is efficient because of the implementation of extensive model reduction and the application of sparse techniques.

\subsubsection{Integrated Adequacy and Security Assessment}

The security assessment of a composite power system involves evaluating system behavior while integrating transient stability as well as cascading sequences after a disturbance. For the correct assessment of system security, the contingency analysis has to be repeated for all significant initial conditions and at different points in time to account for the impact of time-dependent factors, including the transient behavior of generators, the operation of many types of protection schemes, the automatic actions of different controls, and operators' actions. Nowadays, a major effort in the security assessment has been devoted to extending existing adequacy assessment techniques to include the assessment of system security. Other work is based on time domain simulation that achieves the security assessment.

Integration of Transient Stability Limit: A framework that evaluates both the adequacy and security reliability of the system is presented in. In security analysis, the system transient stability limit is identified by comparing the fault clearing time with the critical clearing time. Providing critical clearing time requires a transient stability evaluation. For unstable states, remedial actions are applied, and some indices corresponding to the security evaluation are also provided. The security assessment presented in reference also takes into account the cascading sequences besides the transient stability. System states are classified into nine types: adequate, inadequate, partially adequate, stable, unstable, secure, not secure, marginally adequate, and system collapse states. Based on the classified states, a possible sequence of events after the occurrence of a disturbance is built.

Integration of Security Constraints the security assessment technique presented in is based on the previously described security-constrained adequacy evaluation. In, besides the basic operating constraints that have to be satisfied for the steady- state performance, a transient-performance constraint set that can reflect the transient behavior of a power system when subject to system faults is formed. In, instead of introducing new constraint sets, dynamic system models are suggested for use in determining system operating limits that include security consideration.

Design Contingency Set, a design contingency set (DCS) is set up first. A DCS is a set of contingencies that the system must be able to withstand with acceptable transient performance. Based on the DCS, security limits are derived by ensuring that the system is stable for all DCS contingencies. Security evaluation can be quantified by evaluating the ability of the system to operate within the security limits in the steady state. This method captures the impact of contingencies on security without the need for a transient analysis. However, it may result in the following two problems: (1) because reliability assessment is based on the DCS, it is difficult to determine if the DCS was selected properly and (2) the procedure of determining security limits still requires stability studies.

Time-Domain Simulation a time-domain simulation that obtains the power system response after a disturbance is applied. In the simulation, the dominant dynamic phenomena, i.e., the voltage stability problem, power-frequency control, and the cascading tripping of power system components, are crucial because these processes determine whether new steady-state equilibrium can be reached. The consideration of voltage stability in reliability assessment is addressed in . After the power system reaching a steady state, the procedure of minimizing the overall curtailed load, voltage restoration, and economic dispatching is applied. Because of the complexity of considering transient stability as well as cascading events after contingencies, security assessment incurs an extremely heavy computational burden and has slow convergence.

\subsection{SUMMARY}

Bulk power system reliability assessment includes two aspects: adequacy and security. Of the two, adequacy assessment has been the major concern. The key issue associated with adequacy assessment is the computational burden and the performance of effects analysis to evaluate large-scale systems. Research, which has focused on both analytical and Monte Carlo simulation techniques, has found the former superior to the latter. In addition, a security-constrained adequacy evaluation has recently received more attention, as it is able to simulate actual operational practices that improve the performance of effects analysis procedures. Regarding security assessment, some effort has been devoted to including transient stability analysis and cascading sequences of power system behavior after disturbances occur.

The current bulk power system reliability assessment techniques reveals following facts: (1) the probabilistic modeling method has become the most promising means to represent practical system behavior, (2) the analytical technique is superior to the Monte Carlo simulation technique in performing reliability assessment for large scale bulk power systems, and (3) the contingency selection method and the security- 
constrained adequacy evaluation method are the most effective ways to reduce the system state space and perform contingency effects analysis, respectively. It also indicates that (1) contingency selection and ranking based on the current contingency selection method is prone to misranking, (2) the security-constrained adequacy evaluation method based on the traditional power flow model suffers from the lack of realistic system simulation and slow convergence (even non-convergence) when the system is heavily stressed, (3) no research effort has been devoted to the evaluation of the effects of protection system hidden failures on bulk power system reliability assessment.

\subsection{CONCLUSIONS}

The goal of this paper is centered on adequacy analysis and security reliability evaluation of bulk power system. A comprehensive approach for bulk power system reliability assessment, i.e., the securityconstrained adequacy evaluation (SCAE) methodology, is developed to evaluate the ability of the system in supplying the electric load while satisfying security constraints. Research contributions have been made in following areas:

1. The single phase quadratized power flow (SPQPF) model is applied in the proposed SCAE methodology for bulk power system reliability assessment. Compared with the traditional power flow model, the SPQPF model yields improved contingency selection and ranking accuracy, speeds up the procedure of the effects analysis because of its faster convergence characteristics, and makes the effects analysis more realistic with its ability to model complex load characteristics.

2. An improved critical contingency selection scheme is developed to efficiently identify and rank critical contingencies with high accuracy. Specifically, the system state linearization approach is investigated to reduce the error introduced by the linear approximation in the traditional performance index linearization methods for contingency selection and ranking. The system state linearization approach includes higherorder terms in the performance index calculation procedure to trace nonlinear variations of the performance index for a post-contingency situation and therefore effectively reduce misranking in the contingency selection and ranking procedures.

3. A non-divergent optimal quadratized power flow (NDOQPF) algorithm that performs contingency effects analysis is proposed. Quadratized remedial action models are developed, and the concept of the remedial action control variable is used to represent the availability and amount of system remedial actions. Compared with the traditional power flow solution procedure, the NDOQPF algorithm has the following merits:

(1) It is able to simulate contingencies in a realistic manner to capture the system response including major controls and adjustments. In addition, because of its efficiency, the overall computational effort of SCAE is reasonable;

(2) If a solution exists; it guarantees convergence; if a solution does not exist, such as when multi-level contingencies are considered and the system is severely stressed, it can provide a sub-optimal solution that may include load shedding for the system;

(3) It is applicable to both a regulated and deregulated power system environment. In particular, in a deregulated environment in which the system is more likely to be heavily stressed and may be operated in different power flow patterns from the ones it was originally designed to operate in, the NDOQPF is capable of efficiently solving the ISO/RTO operational model and providing solutions under all conditions.

4. The impact of protection system hidden failures on bulk power system reliability assessment is investigated . A circuit breaker-oriented substation model is introduced to include the detailed substation configuration as well as the protection system scheme in the system network model. In addition, the impact of advanced system real time monitoring technologies on detecting protection system hidden failures is analyzed. Also, a hidden failure effects analysis method is developed to obtain hidden failure outages following any possible initial equipment outages. The security-constrained adequacy evaluation methodology is extended to evaluate contingencies resulting from protection system hidden failures as well as other contingencies resulting from independent and common-mode outages so that the impact of protection system hidden failures on bulk power system reliability assessment is included.

5. The proposed comprehensive bulk power system reliability assessment approach is implemented in a Visual C++ environment using object-oriented techniques and is applied to evaluate the reliability of two reliability test systems. 


\section{References}

[1] M. P. Bhavaraju, R. Billinton, R. E. Brown, J. Endrenyi, W. Li, A. P. Meliopoulos, and C. Singh, IEEE Tutorial on Electric Delivery System Reliability Evaluation, IEEE Power Engineering Society General Meeting, 2005.

[2] R. Billinton and R. N. Allan, Reliability Evaluation of Power Systems, NY: Plenum Press, 1996.

[3] R. Billinton and W. Li, Reliability Assessment of Electric Power Systems Using Monte Carlo Methods, NY: Plenum Press, 1994

[4] J. Endrenyi, Reliability Modeling in Electric Power System, NY: John Wiley \& Sons Ltd., 1978.

[5] A. P. Meliopoulos, R. Kovacs, N. J. Balu, M. Lauby, N. D. Reppen, M. P. Bhavaraju, and R. Billinton, “A Probabilistic Method for Transmission Planning," The $2^{\text {nd }}$ International Symposium on Probability Method Applied in Power Systems, Oakland, USA, September 1988 .

[6] Task Force on Bulk Power System Reliability, "Bulk Power System Reliability Concepts and Applications," IEEE Transactions on Power Systems, Vol. 3, No. 1, pp. 109-117, February 1988.

[7] B. Porretta and E. G. Neudorf, "Conceptual Framework for Evaluation and Interpretation of the Reliability of the Composite Power System," IEEE Transactions on Power Systems, Vol. 10, No. 2, pp. 1094-1103, May 1995.

[8] R. Billinton, L. Salvaderi, J. D. McCalley, H. Chao, T. Seitz, R. N. Allen, J. Odom, and C. Fallon, "Reliability Issues in Today's Electric Power Utility Environment," IEEE Transactions on Power Systems, Vol. 12, No. 4, pp. 1708-1714, November 1997.

[9] K. N. Zadeh, R. C. Meyer, and G. Cauley, "Practices and New Concepts in Power System Control," IEEE Transactions on Power Systems, Vol. 11, No. 1, pp. 3-10, February 1996.

[10] Z. Alaywan and J. Allen, "California Electric Restructuring," IEEE Transactions on Power Systems, Vol. 13, No. 4, pp. 1445-1452, November 1998.

[11] J. Katzel, “Managing Energy in a Deregulated Environment: Opportunity or Chaos?” Plant Engineering, pp. 36-42, January 1999.

[12] H. B. Puttgen, D.R. Volzka, and M. I. Olken, "Restructuring and Re-regulation of the US Electric Utility Industry," IEEE Power Engineering Review, Vol. 21, No. 2, pp. 8-10, February 2001.

[13] Y. V. Makarov and R. C. Hardiman, "Risk, Reliability, Cascading and Restructuring," IEEE Power Engineering Society General Meeting, Vol. 3, pp. 1417-1429, July 2003.

[14] W. J. Lee and C. H. Lin, "Utility Deregulation and Its Impact on the Industrial Power Systems," Industrial and Commercial Power Systems Technical Conference, pp. 217-222, May 1997.

[15] L. H. Fink and P. J. M. V. Son, “On System Control within a Restructured Industry,” IEEE Transactions on Power Systems, Vol. 18, No. 2, pp. 611-616, May 1998.

[16] J. W. M. Cheng, D. T. McGillis, and F. D. Galiana, "Power System Reliability in a Deregulated Environment," Conference on Electrical and Computer Engineering, Vol. 2, pp. 765-768, Mar. 2000.

[17] J. De La Ree, Y. Liu, L. Mili, A. G. Phadke, and L. Dasilva, "Catastrophic Failures in Power Systems, Cause, Analysis, and Countermeasures," IEEE Proceedings, Vol. 93, No. 5, pp. 956-964, 2005.

[18] R. Allan and R. Billinton, "Power System Reliability and its Assessment. Part 1 Background and Generating Capacity," Power Engineering Journal, Vol. 6, No. 4, pp. 191-196, July 1992.

[19] R. Allan and R. Billinton, "Power System Reliability and its Assessment. Part 2 Composite generation and transmission systems," Power Engineering Journal, Vol. 6, No. 6, pp. 291-297, November 1992.

[20] B. L. Silverstein and D. M. Porter, "Contingency Ranking for Bulk System Reliability Criteria," IEEE Transactions on Power Systems, Vol. 7, No. 3, pp. 956-964, August 1992.

[21] B. E. Arporn and A. Karunanoon, "Reliability Evaluation in Electrical Power Generation with Uncertainty Modeling by Fuzzy Number," IEEE Power Engineering Society Summer Meeting, Vol.4, pp. 2051-2056, July 2000.

[22] J. T. Saraiva, "Reliability Evaluation of Generation/Transmission Power Systems Including Fuzzy Data," 1996 IEEE International Symposium on Circuits and Systems, Vol. 1, pp. 609-612, May 1996.

[23] J. T. Saraiva, V. Miranda, and L. M. V. G. Pinto, "Generation/Transmission Power System Reliability Evaluation by Monte-Carlo Simulation Assuming a Fuzzy Load Description,” IEEE Transactions on Power Systems, Vol. 11, No. 2, pp. 690-695, May 1996.

[24] J. T. Saraiva and A. V. Sousa, "New Advances in Integrating Fuzzy Data in Monte Carlo Simulation to Evaluate Reliability Indices of Composite Power Systems," Electrotechnical Conference, Mditerranean, Vol. 2, pp. 1084-1088, May 1998.

[25] V. Miranda and J. T. Saraiva, "Fuzzy Modeling of Power System Optimal Load Flow," IEEE Transactions on Power Systems, Vol. 7, No. 2, pp. 843-849, May 1992.

[26] J. T. Saraiva, V. Miranda, and L. M. V. G. Pinto, "Impact on Some Planning Decisions From a Fuzzy Modeling of Power Systems," IEEE Transactions on Power Systems, Vol. 9, No. 2, pp. 819-825, May 1994.

[27] M. T. Schilling, R. Billinton, A. M. L. D. Silva, and M. A. E. Kady, "Bibliography on Composite System Reliability 1964-1988," IEEE Transactions on Power Systems, Vol. 4, No. 3, pp. 1122-1132, August 1989.

[28] R. N. Allan, R. Billinton, S. M. Shahidehpour, and C. Singh, "Bibliography on the Application of Probability Methods in Power System Reliability Evaluation 1982-1987," IEEE Transactions on Power Systems, Vol. 3, No. 4, pp. 1555-1564, November 1988.

[29] R. N. Allan, R. Billinton, A. M. Breipohl, and C. H. Grigg, "Bibliography on the Application of Probability Methods in Power System Reliability Evaluation 1987-1991," IEEE Transactions on Power Systems, Vol. 9, No. 1, pp. 41-49, February 1994.

[30] R. N. Allan, R. Billinton, A. M. Breipohl, and C. H. Grigg, "Bibliography on the Application of Probability Methods in Power System Reliability Evaluation 1992-1996," IEEE Transactions on Power Systems, Vol. 14, No. 1, pp. 51-57, February 1999.

[31] R. Billinton, M. F. Firuzabad, and L. Bertling, "Bibliography on the Application of Probability Methods in Power System Reliability Evaluation 1996-1999," IEEE Transactions on Power Systems, Vol. 14, No. 1, pp. 595-602, February 1999.

[32] E. Liu, “Technology Integration-Computing and Analytical Methods,” Power Engineering Society Summer meeting, Vol. 2, July 1999.

[33] EPRI Report EL-2526, “Transmission System Reliability Methods,” July 1982.

[34] EPRI Report EL-5291, "Reliability Evaluation for Large-Scale Bulk Transmission Systems," Comparative Evaluation, Method Development, and Recommendation, Vol. 1, January 1988.

[35] R. Billinton and A. Sankarakrishnan, "A Comparison of Monte Carlo Simulation Techniques for Composite Power System Reliability Assessment," Proceedings of IEEE Conference on Communications, Power, and Computing, Vol.1, pp. 145-150, May 1995.

[36] M. E. Khan and R. Billinton, “A Hybrid Model for Quantifying Different Operating States of Composite Power Systems,” IEEE Transactions on Power Systems, Vol. 7, No. 1, pp. 187-193, February 1992.

[37] Y. Guo, Y. Xi, K. Xiao, and H. Yang, "Composite System Reliability Evaluation Based on Monte-Carlo Simulation Combined with Outages Screening,” IEEE Transactions on Power Systems, Vol. 14, No. 1, pp. 785-790, May 1999.

[38] X. Luo, C. Singh, and A. D. Patton, "Power System Reliability Evaluation Using Self Organizing Map," IEEE Power Engineering Society Winter Meeting, Vol. 2, pp. 1103-1108, January 2000. 
[39] R. Billinton and E. Khan, “A Security Based Approach to Composite Power System Reliability Evaluation,” IEEE Transactions on Power Systems, Vol. 7, No. 1, pp. 65-72, February 1992.

[40] R. Billinton and W. Zhang, "State Extension in Adequacy Evaluation of Composite Power Systems --- Concept and Algorithm," Electric Power Systems Research, Vol. 14, pp.189-195, 1998.

[41] R. Billinton and W. Zhang, "State Extension for Adequacy Evaluation of Composite Power Systems --- Applications," IEEE Transactions on Power Systems, Vol. 15, No. 1, pp. 427-432, February 2000.

[42] R. Billinton and S. K. Agarwal, "Examination of severe contingencies in a small area of a large composite power system using adequacy equivalent," IEE Proceedings, Vol. 137, No. 2, pp.107-114, March 1990.

[43] S. Kumar and R. Billinton, "Adequacy Equivalents in Composite Power System Evaluation," IEEE Transactions on Power Systems, Vol. 3, No. 3, pp. 1167-1173, August 1998.

[44] R. Billinton and W. Zhang, "Enhanced Adequacy Equivalent for Composite Power System Reliability Evaluation," IEE Proc.-Gener. Transm. Distrib., Vol. 143, No.5, September 1996.

[45] W. Zhang and R. Billinton, "Application of an Adequacy Equivalent Method in Bulk Power System Reliability Evaluation," IEEE Transactions on Power Systems, Vol. 13, No. 2, pp. 661-666, May 1998.

[46] H. A. M. Maghraby and R. N. Allan, "Application of DC Equivalents to the Reliability Evaluation of Composite Power Systems," IEEE Transactions on Power Systems, Vol. 14, No. 1, pp. 355-361, February 1999.

[47] J. Zaborszky and F. W. Whang, "Fast Contingency Evaluation Using Concentric Realization," IEEE Transactions on Power Apparatus and Systems, Vol. PAS-99, No. 1, pp. 28-36, January/February 1980.

[48] V. Brandwajn, "Efficient Bounding Method for Linear Contingency Analysis," IEEE Transactions on Power Systems, Vol. PWRS-3, No. 1, pp. 38-43, February 1988.

[49] V. Brandwajn and M. G. Lauby, "Complete Bounding for AC Contingency Analysis,” IEEE Transactions on Power Systems, Vol. PWRS-4, No. 2, pp. 724-729, May 1990

[50] A. P. S. Meliopoulos and C. Cheng, “A Hybrid Contingency Selection Method," Proceedings of the $10^{\text {th }}$ Power System Computation Conference, Austria, pp. 605-612, August 1994.

[51] S. Kang and A. P. S. Meliopoulos, "Analytical Approach for the Evaluation of Actual Transfer Capability in a Deregulated Environment," Proceedings of the 32 ${ }^{\text {nd }}$ Annual North American Power Symposium, 1999.

[52] A. P. Sakis Meliopoulos, Power System Modeling, Analysis and Control, Georgia Institute of Technology, 2002.

[53] G. C. Ejebe and B. F. Wollenberg, "Automatic Contingency Selection," IEEE Transactions on Power Apparatus and Systems, Vol. PAS-98, No.1, pp.92-104, January/February 1979.

[54] A. P. Sakis Meliopoulos, C. S. Cheng, and F. Xia, "Performance Evaluation of Static Security Analysis Methods," IEEE Transactions on Power Systems, Vol. 9, No. 3, pp. 1441-1449, August 1994.

[55] G. Irisarri, A. M. Sasson, and D. Levner, "Automatic Contingency Selection for On-Line Security Analysis-Real Time Tests," IEEE Transactions on Power Apparatus and Systems, Vol. PAS-98, No.5, pp.1552-1559, September/October 1979.

[56] A. J. Wood and B. F. Wollenberg, Power Generation, Operation, and Control, John Wiley \& Sons, Inc. 1984.

[57] IEEE Committee Report, "Parallel Processing in Power Systems Computation," IEEE Transactions on Power Systems, Vol. 7, No. 2, pp. 629-638, May 1992.

[58] C. L. T. Borges, D. M. Falcao, J. C. O. Mello, and A. C. G. Melo, "Composite Reliability Evaluation by Sequential Monte Carlo Simulation on Parallel and Distributed Processing Environment," IEEE Transactions on Power Systems, Vol. 16, No. 2, pp. 203-209, May 2001.

[59] C. Lemaitre and B. Thomas, "Two Applications of Parallel Processing in Power System Computation," IEEE Transactions on Power Systems, Vol. 11, No. 1, pp. 246-253, February 1996.

[60] M. J. Teixeira, H. J. C. P. Pinto, M. V. F. Pereira, and M. F. McCoy, "Developing Concurrent Processing Applications to Power System Planning and Operations," IEEE Transactions on Power Systems, Vol. 5, No. 2, pp. 659-664, May 1990.

[61] N. Gubbala and C. Singh, "Models and Considerations for Parallel Implementation of Monte Carlo Simulation Methods for Power System Reliability Evaluation,” IEEE Transactions on Power Systems, Vol. 10, No. 2, pp. 779-787, May 1995.

[62] D. J. Boratynska-Stadnicka, M. G. Lauby, and J. E. V. Ness, "Converting an Existing Computer Code to a Hypercube Computer," Power Industry Computer Application Conference, pp. 394-399, May 1989.

[63] C. Singh and J. Mitra, "Composite System Reliability Evaluation using State Space Pruning," IEEE Transactions on Power System s, Vol. 12, No. 1, pp. 471-479, February

[64] D. P. Clancy, G. Gross, and F. F. Wu, "Probabilistic Flows for Reliability Evaluation of Multi-area Power System Interconnections," Electric Power \& Energy Systems, Vol. 5, No. 2, pp.101-114, April 1983.

[65] C. Singh, and Z. Deng, "A New Algorithm for Multi-Area Reliability Evaluation-Simultaneous Decomposition Simulation Approach,” Electric Power \& Energy Systems, Vol. 21, No. 2, pp.129-136, April. 1983.

[66] Z. Deng and C. Singh, "A New Approach to Reliability Evaluation of Interconnected Power Systems Including Planned Outages and Frequency Calculations," IEEE Transactions on Power Systems, Vol. 7, No. 2, pp. 734-743, May 1992.

[67] J. Mitra and C. Singh, "Incorporating the DC Load Flow Model in the Decomposition-Simulation Method of Multi-Area Reliability Evaluation,” IEEE Summer Power Meeting, July 1995.

[68] J. Mitra, Models for Reliability Evaluation of Multi-Area and Composite, Ph.D. Dissertation, Texas A\&M University, TX, 1997.

[69] J. Mitra and C. Singh, "Pruning and Simulation for Determination of Frequency and Duration Indices of Composite Power Systems," IEEE Transactions on Power Systems, Vol. 14, No. 3, pp. 899-905, August 1999.

[70] K. Bae and J. S. Thorp, “An Importance Sampling Application: 179 Bus WSCC System under Voltage Based Hidden Failures and Relay Mis-operations,” International Conference on System Science, January 1997.

[71] A. G. Phadke and J. S. Thorp, "Expose Hidden Failures to Prevent Cascading Outages," IEEE Computer Application in Power, pp. 20-23, July 1996.

[72] M. V. F. Pereira, M. E. P. Maceira, and L. M. V. G. Pinto, "Combining Analytical Models and Monte-Carlo Techniques in Probabilistic Power System Analysis,” IEEE Transactions on Power Systems, Vol. 7, No. 1, pp. 265-272, February 1992.

[73] M. E. Khan and R. Billinton, "A Hybrid for Quantifying Different Operating States of Composite Power Systems," IEEE Transactions on Power Systems, Vol. 7, No. 1, pp. 187-193, February 1992.

[74] A. C. G. Melo, G. C. Oliverira, M. Morozaowski, and M. V. F. Pereira, "A Hybrid Algorithm for Monte Carlo/Enumeration Based Composite Reliability Evaluation," Third International Conference on Probabilistic Methods Applied to Electric Power Systems, pp.70-74, July 1991. 